\title{
Two sisters reveal autosomal recessive inheritance of epidermodysplasia verruciformis: a case report
}

\author{
Rui Yoshida ${ }^{1}$, Toshihiko Kato ${ }^{2}$, Masahiko Kawase ${ }^{3}$, Mariko Honda ${ }^{3}$ and Tsuyoshi Mitsuishi ${ }^{*}$
}

\begin{abstract}
Background: Epidermodysplasia verruciformis is a rare genodermatosis characterized by a unique susceptibility to cutaneous human papillomaviruses infection. Most patients show autosomal recessive patterns of inheritance.

Case presentation: We report a case of two sisters with clinically epidermodysplasia verruciformis specific lesions on the face, neck, trunk, and extremities. PCR analysis indicated the presence of human papillomavirus type 5 in the lesions. Electron microscopic examination showed viral-like particles in keratinocyte nuclei and the stratum corneum of the epidermodysplasia verruciformis lesions. In addition, we examined the EVER1 and EVER2 genes using eight different primer pairs without finding any nonsense or frameshift mutations in the gDNA from lymphocytes of the elder sister.

Conclusions: In this report, the patient's parents did not have epidermodysplasia verruciformis lesions or a consanguineous marriage. EV did not develop in the elder sister until five years of age, so the parents did not perceive $\mathrm{EV}$ as an inherited disease. The probability that EV developed in both sisters was only $6.25 \%$. Thus, it is rare for both sisters to develop epidermodysplasia verruciformis lesions considering that the parents were presumed to be carriers and the disease reveal an autosomal recessive pattern of inheritance.
\end{abstract}

Keywords: Epidermodysplasia verruciformis, Hereditary pattern, Human papillomavirus, EVER1 and EVER2 genes, Electron microscopic examination

\section{Background}

Epidermodysplasia verruciformis (EV) is an uncommon cutaneous disorder characterized by persistent infection with beta-human papillomavirus (HPV) and a combination of flat wart-like lesions, pityriasis versicolor-like lesions, hypopigmented macules, or other erratic skin lesions. These lesions are mainly located on the face, neck, and extremities [1,2]. Most patients demonstrate autosomal recessive patterns of inheritance, although some exhibit X-linked recessive or autosomal dominant inheritance [3,4]. Cutaneous carcinomas in situ or invasive carcinomas, which are usually of the Bowen's disease-type, generally appear in a high percentage of patients, before the age of $40[1,2,5]$. More than 40 betapapillomaviruses have been identified in patients with EV [6]. Among them, HPV5 and HPV8 are primarily involved in the malignant transformation associated with EV. HPV9 is also often involved in EV [1,2].

\footnotetext{
* Correspondence: tmitsu@tymc.twmu.ac.jp

${ }^{4}$ Department of Dermatology, Tokyo Women's Medical University Yachiyo Medical Center, 477-96, Ohwada-Shinden, Yachiyo 276-8524, Chiba, Japan Full list of author information is available at the end of the article
}

The EVER1 and EVER2 genes were found to be responsible for $\mathrm{EV}$ in 2002 [7]. EVER1 and EVER2 are also referred to as TMC6 and TMC8, respectively, and are located on chromosomes 17q25 [7]. Various mutations in the EVER1 and/or EVER2 genes reportedly contribute to HPV-associated EV [8-10]. In this study, we report a case of two sisters with both EV and HPV5 infections whose parents did not have EV lesions. We observed viral-like particles in the lesions by electron microscopic examination. We also assessed the mutational status of the EVER1 and EVER2 genes from extracted blood cells using PCR with eight different primers.

\section{Case presentation}

\section{Case 1}

A 29-year-old female has had asymptomatic, slightly erythematous, and hypopigmented pityriasis versicolorlike lesions on her face, neck, trunk, and extremities since the age of five as well as multiple brownish-black, hyperkeratotic, papular plaque, wart-like lesions on the extremities. The lesions progressively increased in number and size with age (Figure 1A, B). No abnormalities were 


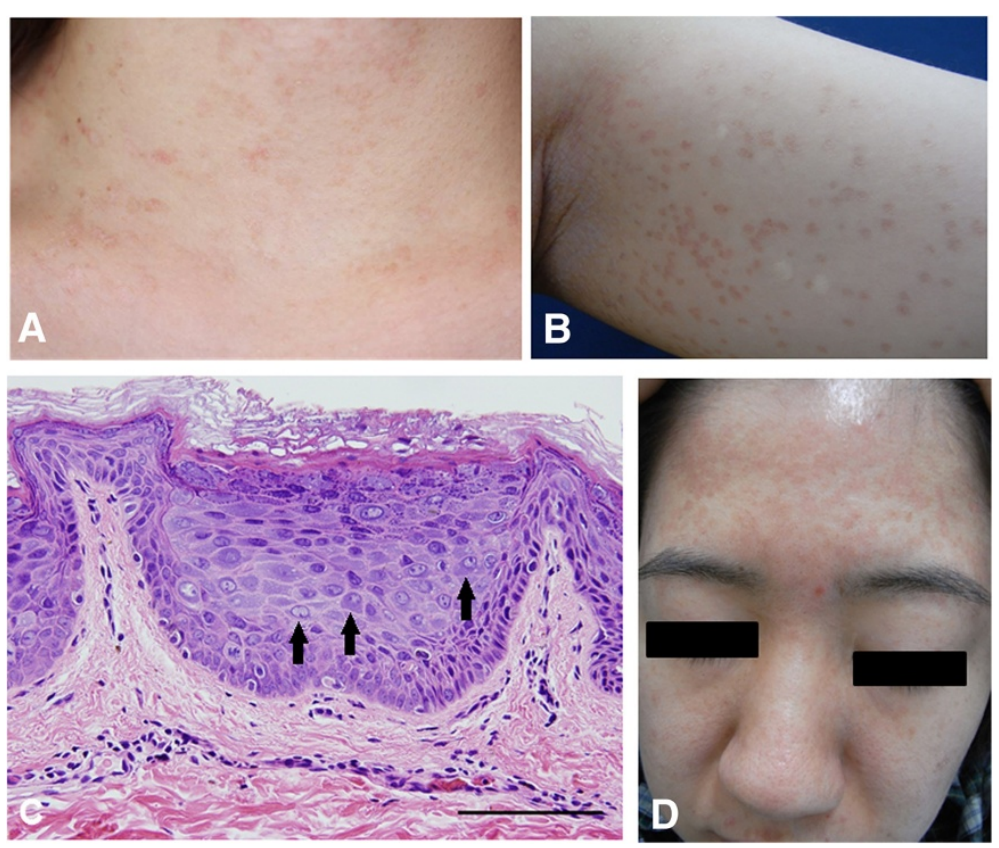

Figure 1 Clinicohistological findings in two sisters. (A) Asymptomatic, slightly erythematous, and hypopigmented pityriasis versicolor-like lesions on the neck in the elder sister. (B) Left upper arm of the elder sister showing numerous round pigmented macules with slight scale. (C) Histological findings show epidermal hyperplasia and distinct homogenous intracytoplasmic inclusion bodies in the large clear cells of the epidermis (arrows); scale bar $=100 \mu \mathrm{m}$. (D) Multiple brown, flat wart-like lesions on the face of the younger sister.

observed upon examination of the hair, nails, mucosal membranes, and other systems, including abdominal echography and chest X-ray. Histopathology of a flat wart-like lesion showed marked hyperkeratosis, mild acanthosis, and the presence of distinct homogeneous intracytoplasmic inclusion bodies in the large clear cells of the epidermis (Figure 1C). Additional systemic examinations and laboratory investigations, including an HIV test, were all normal. Topical 5-fluorouracil (5-FU) and imiquimod $5 \%$ cream were applied to the small pityriatic or flat wart-like lesions for three days per week at Jikei University School Hospital and Nippon Medical School Hospital. The lesions gradually decreased in size and number. She has been applying ultraviolet (UV) blocker most days and is being followed by Nippon Medical School Hospital.

\section{Case 2}

The 23-year-old sister of case 1 has had similar cutaneous lesions since the age of six. Multiple asymptomatic, erythematous, pityriasis versicolor-like lesions and flat wartlike lesions were found on the face, trunk, and extremities (Figure 1D). These lesions also increased progressively in number and size. A biopsy of the EV lesions showed focal cellular atypia in the flat wart-like lesions with abnormal mitotic figures in the large clear cells of the epidermis. Topical 5-FU and imiquimod 5\% cream were prescribed for application twice daily on the lesions. She has been applying UV blocker almost every day. Interestingly, neither parents of the two patients showed EV lesions, but both were carriers.

\section{Genetic investigations}

We performed PCR using two different primers, CP65/ 70 and SK, to determine the prevalence of HPV infection $[11,12]$. Frozen tissues from the EV cutaneous lesions of both patients were digested with proteinase $\mathrm{K}$ (100 mg/ml), and genomic DNA (gDNA) was extracted with phenol-chloroform-isoamyl alcohol. The gDNA was then precipitated with ethanol as previously described [12]. The primer sets amplified DNA products from alpha-, beta-, and gamma-HPVs. The PCR products were sequenced with an ABI model 3730xl automated DNA sequencer (Applied Biosystems, Foster City, CA, USA) according to the manufacturer's instructions. The result of the PCR analysis indicated that HPV5 infection was present in the flat wart-like lesions and pityriasis versicolor-like lesions from the lesions in both sisters.

After informed consent was obtained, gDNA from the peripheral blood of the elder sister was acquired according to the manufacturer's instructions. The presence of EVER1 and EVER2 mutations was analyzed by PCR with six known primers [8-10] and two new primers, which were designed against GenBank sequences (NM_152468) for TMC8/EVER2. The new primers used for amplification of the gDNAs were as follows: EVER2, exon 8-1 F, 5'- 
GTG GAG CTG GAG GAG GG-3'; exon 8-1R, 5'-TCC TTG TTG TCC TGT GAG TAC TTG-3'; exon 8-2 F, 5'GTT TCC TGC ACC CTT TCC TC-3'; exon 8-2R, 5'TAC TTG GTA GCC CAG AAG ATG G-3'. The PCR products from the two new primers were $170 \mathrm{bp}$ and $211 \mathrm{bp}$ in length, respectively. The PCR products were run on $2 \%$ agarose gels and purified with the QIAquick Gel Extraction Kit (Qiagen, Hilden, Germany). Sequencing analysis was carefully performed in both directions and compared with the known sequences registered in GenBank. We did not identify any nonsense or frameshift mutations in any of the eight target bands.

\section{Electron microscopic examination}

Electron microscopy was performed on a lesion from the skin of the elder sister. The specimens were fixed with $2.5 \%$ glutaraldehyde and postfixed with $1 \%$ osmium tetroxide. The samples were then dehydrated through a graded alcohol series and embedded in Epon 812. Ultrathin sections were cut using an Ultracut N Ultramicrotome (Reihert-Nissei, Tokyo, Japan) and were stained with uranyl acetate and lead citrate. The sections were then examined by electromicroscope (Hitachi H-7500; Hitachi, Tokyo, Japan). The results of the electron microscopy showed typical virus-like particles with crystalline arrays filling the nuclei of the keratinocyte cells in the upper epidermis and stratum corneum. Large, clear dysplastic cells were also observed in the flat wart-like lesions (Figure 2).

\section{Discussion}

Malignant cutaneous lesions in patients with EV are preferentially located on sun-exposed areas. The development of malignant transformation in EV patients is primarily associated with HPV5 and HPV8 infection. PCR analysis indicated that HPV5 infection was present in flat wart-like lesions and pityriasis versicolor-like lesions of our patients; however, malignant cutaneous lesions were not found in sun-exposed areas. Most patients have autosomal recessive inheritance patterns for EV. However, a small number of patients show X-linked recessive or autosomal dominant inheritance [3,4]. The parents of the patients in our study were presumed to be carriers, but did not develop EV lesions. In addition, the parents did not form a consanguineous marriage. Based on autosomal recessive patterns of inheritance, we would expect $25 \%$ of children to develop EV lesions. In this family, these sisters are the only offspring, and the mother did not have a history of miscarriage. EV did not develop in the elder sister until five years of age. Thus, the parents did not identify $E V$ as a disease of inheritance.

The EVER1 and EVER2 genes associated with EV belong to the transmembrane channel-like $(T M C)$ gene family and are called TMC6 and TMC8, respectively.

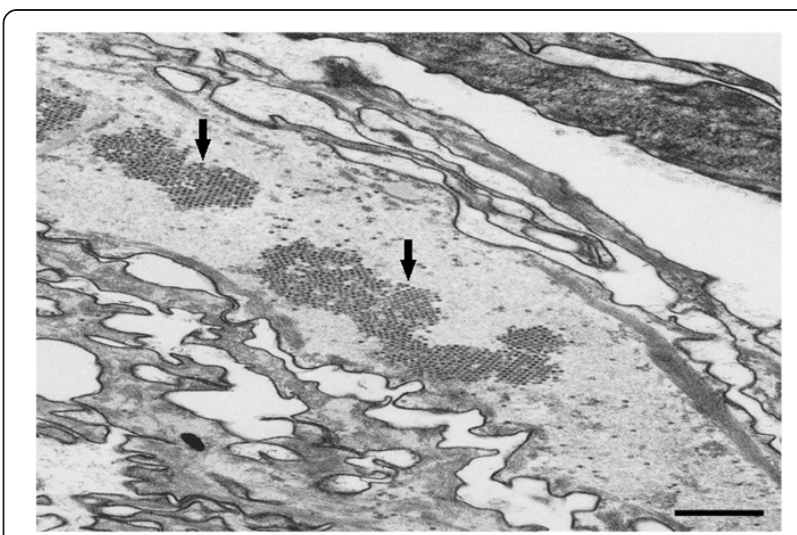

Figure 2 Electron microscopy image showing typical virus-like particles with crystalline array (arrows) filling the stratum corneum; scale bar $=1 \mu \mathrm{m}$.

The albuminous functions encoded by EVER1 and EVER2 remain unknown. The presence of EVER gene mutations in patients with EV suggests that beta-HPV infection is linked to the progression of non-melanoma skin cancer. Some reports indicate the relationship between mutations in the EVER2 gene and the risk of cutaneous squamous cell carcinoma [13,14]. After careful examination for the presence of EVER1 and EVER2 genes by PCR followed sequence analysis in both directions, we did not observe any nonsense or frameshift mutations in any of the target bands. Although there is no curative therapy for $\mathrm{EV}$, protection from UV light and treatment of EV-specific cutaneous lesions is necessary from an early stage. Nonsurgical approaches include topical imiquimod, 5-FU, vitamin D3, cimetidine, systemic retinoids, and interferon [14-18]. Some reports show success with topical imiquimod in immunocompetent patients with squamous cell carcinoma in situ $[14,19]$. In this study, imiquimod $5 \%$ cream was applied once per day and three days per week for flat wart-like lesions. Oral retinoids $(0.5 \mathrm{mg} / \mathrm{kg})$ were also prescribed. Cutaneous lesions decreased in size after a short period; however, relapse of the cutaneous lesions of patients occurred after 1 year, suggesting that the therapy was not effective. Importantly, both patients applied UV blocker daily.

\section{Conclusions}

Most EV patients show autosomal recessive patterns of inheritance; although, some patients can exhibit X-linked recessive or autosomal dominant inheritance. In the two cases described here, two sisters with EV had parents without EV lesions that were are not in a consanguineous marriage. Furthermore, the mother does not have a history of miscarriage. EV did not develop in the elder sister until five years of age. The probability of EV developing in both sisters was only $6.25 \%$. Thus, it is rare for both sisters to develop EV lesions, despite that both parents were 
presumed to be carriers and the disease showed an autosomal recessive pattern of inheritance. No nonsense or frameshift mutations in the EVER2 gene were identified using two new primers, however, there may be some risk of developing cutaneous squamous cell carcinoma from benign EV lesions. Long-term follow-up will be necessary for both sisters.

\section{Consent}

Written informed consent was obtained from the patients for publication of this Case report and any accompanying images. A copy of the written consent is available for review by the Editor of this journal.

\section{Abbreviations}

EV: Epidermodysplasia verruciformis; HPV: Human papillomavirus; UV: Ultraviolet; gDNA: Genomic DNA.

\section{Competing interests}

The authors declared that they have no competing interests.

\section{Authors' contributions}

$\mathrm{RY}, \mathrm{MK}, \mathrm{MH}$, and TM analysed clinical and pathological data, including those from patient follow up. TK, and TM performed laboratory work and helped with data analysis. RY, and TM drafted the manuscript and designed the case-study. All authors read and approved the final manuscript.

\section{Acknowledgements}

We thank Dr. Ikuroh Ohsawa (Department of Biological Process of Aging, Tokyo Metropolitan Institute of Gerontology) for reading our manuscript.

\section{Author details}

'Department of Dermatology, Nippon Medical School, 1-1-5, Sendagi, Bunkyo-ku, Tokyo 113-8603, Japan. ${ }^{2}$ The Research Institute of Vaccine Therapy for Tumors and Infectious Diseases, Nippon Medical School, 1-1-5, Sendagi, Bunkyo-ku, Tokyo 113-8603, Japan. ${ }^{3}$ Department of Dermatology, The Jikei University School of Medicine, 3-25-8, Nishi Shinbashi, Minato-ku 105-8461, Tokyo, Japan. ${ }^{4}$ Department of Dermatology, Tokyo Women's Medical University Yachiyo Medical Center, 477-96, Ohwada-Shinden, Yachiyo 276-8524, Chiba, Japan.

Received: 11 April 2014 Accepted: 16 July 2014

Published: 21 July 2014

\section{References}

1. Orth G, Jablonska S, Favre M, Croissant O, Jarzabek-Chorzelska M, Rzesa G: Characterization of two types of human papillomaviruses in lesions of epidermodysplasia verruciformis. Proc Natl Acad Sci U S A 1978, 75:1537-1541

2. Orth G, Jablonska S, Jarzabek-Chorzelska M, Obalek S, Rzesa G, Favre M, Croissant $\mathrm{O}$ : Characteristics of the lesions and risk of malignant conversion associated with the type of human papillomavirus involved in epidermodysplasia verruciformis. Cancer Res 1979, 39:1074-1082.

3. Androphy E, Dvoretzky I, Lowy DR: X-linked inheritance of epidermodysplasia verruciformis. Genetic and virologic studies of a kindred. Arch Dermatol 1985, 121:864-868.

4. McDermott DF, Gammon B, Snijders PJ, Mbata I, Phifer B, Howland Hartley A, Lee CC, Murphy PM, Hwang ST: Autosomal dominant epidermodysplasia verruciformis lacking a known EVER1 or EVER2 mutation. Pediatr Dermatol 2009, 26:306-310.

5. Mitsuishi T, Ohara K, Suzuki T, Mochizuki T, Kaneko T, Kawana S: Epidermodysplasia verruciformis with keratoacanthoma, Bowen's disease and squamous cell carcinoma: isolation of high-risk types of HPV 5 and unknown type of human papillomavirus. J Eur Acad Dermatol Venereol 2008, 22:1126-1127.
6. Bernard HU, Burk RD, Chen Z, van Doorslaer K, zur Hausen H, de Villiers EM: Classification of papillomaviruses (PVs) based on $189 \mathrm{PV}$ types and proposal of taxonomic amendments. Virology 2010, 401:70-79.

7. Ramoz N, Rueda LA, Bouadjar B, Montoya LS, Orth G, Favre M: Mutations in two adjacent novel genes are associated with epidermodysplasia verruciformis. Nat Genet 2002, 32:579-581.

8. Zuo YG, Ma D, Zhang Y, Qiao J, Wang B: Identification of a novel mutation and a genetic polymorphism of EVER1 gene in two families with epidermodysplasia verruciformis. J Dermatol Sci 2006, 44:153-159.

9. Tate G, Suzuki T, Kishimoto K, Mitsuya T: Novel mutations of EVER1/TMC6 gene in a Japanese patient with epidermodysplasia verruciformis. I Hum Genet 2004, 49:223-225.

10. Arnold AW, Burger B, Kump E, Rufle A, Tyring SK, Kempf W, Häusermann P, Itin PH: Homozygosity for the c.917A $\rightarrow$ T (p.N306l) polymorphism in the EVER2/TMC8 gene of two sisters with epidermodysplasia verruciformis Lewandowsky-Lutz originally described by Wilhelm Lutz. Dermatology 2011, 222:81-86.

11. Berkhout RJ, Tieben LM, Smits HL, Bavinck JN, Vermeer BJ, ter Schegget J: Nested PCR approach for detection and typing of epidermodysplasia verruciformis-associated human papillomavirus types in cutaneous cancers from renal transplant recipients. J Clin Microbiol 1995, 33:690-695.

12. Sasagawa T, Mitsuishi T: Novel polymerase chain reaction method for detecting cutaneous human papillomavirus DNA. J Med Virol 2012, 84:138-144.

13. Patel AS, Karagas MR, Pawlita M, Waterboer T, Nelson HH: Cutaneous human papillomavirus infection, the EVER2 gene and incidence of squamous cell carcinoma: a case-control study. Int J Cancer 2008, 122:2377-2379.

14. Berthelot C, Dickerson MC, Rady P, He Q, Niroomand F, Tyring SK, Pandya AG: Treatment of a patient with epidermodysplasia verruciformis carrying a novel EVER2 mutation with imiquimod. J Am Acad Dermatol 2007, 56:882-886.

15. Anadolu R, Oskay T, Erdem C, Boyvat A, Terzi E, Gürgey E: Treatment of epidermodysplasia verruciformis with a combination of acitretin and interferon alfa-2a. J Am Acad Dermatol 2001, 45:296-299.

16. Gubinelli E, Posteraro P, Cocuroccia B, Girolomoni G: Epidermodysplasia verruciformis with multiple mucosal carcinomas treated with pegylated interferon alfa and acitretin. J Dermatolog Treat 2003, 14:184-188.

17. Micali G, Nasca MR, Dall'Oglio F, Musumeci ML: Cimetidine therapy for epidermodysplasia verruciformis. J Am Acad Dermatol 2003, 48:S9-S10.

18. Hayashi J, Matsui C, Mitsuishi T, Kawashima M, Morohashi M: Treatment of localized epidermodysplasia verruciformis with tacalcitol ointment. Int J Dermatol 2002, 41:817-820.

19. Heratizadeh A, Völker B, Kupsch E, Wichmann K, Kapp A, Werfel T: Successful symptomatic treatment of epidermodysplasia verruciformis with imiquimod 5\% cream. Hautarzt 2010, 61:1052-1055.

doi:10.1186/1471-5945-14-12

Cite this article as: Yoshida et al.: Two sisters reveal autosomal recessive inheritance of epidermodysplasia verruciformis: a case report. BMC Dermatology 2014 14:12.

\section{Submit your next manuscript to BioMed Central and take full advantage of:}

- Convenient online submission

- Thorough peer review

- No space constraints or color figure charges

- Immediate publication on acceptance

- Inclusion in PubMed, CAS, Scopus and Google Scholar

- Research which is freely available for redistribution 